\title{
Medikamentenmangel im Pharmaland Schweiz
}

Thomas Cerny ${ }^{a}$,

Dieter Conen ${ }^{b}$

a Prof. Dr. med., Präsident Krebsforschung Schweiz, Vorstandsmitglied Krebsliga Schweiz, Chefarzt Onkologie/ Hämatologie, Kantonsspital St. Gallen

b Prof. Dr. med., Präsident Stiftung Patientensicherheit
Korrespondenz:

Prof. Dr. med. Thomas Cerny

Dept. Innere Medizin,

Onkologie Hämatologie

Kantonsspital

CH-9007 St. Gallen

Tel. 0714941062

Fax 0714942878

thomas.cerny[at]kssg.ch

Dipl.-Kffr. Roswitha Scheidweiler RS Medical Consult GmbH Frauenberg 1

D-97980 Bad Mergentheim

Tel. 0049793152612

Fax 00497931561226

info[at]rsmedicalconsult.com

www.rsmedicalconsult.com
Noch bis vor kurzen war eine solche Affiche undenkbar, dass wir in der Schweiz, dem Pharmaland par excellence, auf unersetzliche und lebensrettende Medikamente nicht jederzeit, pünktlich und in verlässlicher Qualität zurückgreifen könnten. Nun sind auch diese guten alten Zeiten vorbei. Wir lesen fast täglich, dass sich Spitalapotheker abmühen, für ihre Spitäler die notwendigen Medikamente, insbesondere intravenöse Krebsmittel, Antibiotika und Anästhetika, zu beschaffen. Solche verstörenden Entwicklungen sind in den USA bereits etwas länger zu beobachten. So wird dort bereits hochgerechnet, wie viele Patienten wohl an den Folgen von Medikamentenengpässen zu Schaden gekommen sind. Parlamentarische Hearings in Washington legen davon beredt Zeugnis ab.

Es lohnt sich, rechtzeitig zu analysieren, welche Ursachen vorliegen und welche Folgen resultieren und welche wirksamen Massnahmen getroffen werden müssen

\section{Was sind mögliche Ursachen des Medikamen- tenmangels oder Medikamentenausfalls?}

- Der Arzneimittelmarkt ist heute nicht mehr regional, sondern globalisiert und ist in den Produktions- und Absatzkanälen kaum mehr überschaubar. Substanzen und Komponenten, gerade auch aus Rohstoffen für die Medikamentenherstellung, sind immer mehr in den Händen von wenigen globalen Anbietern - zunehmend auch ausserhalb der westlichen Länder, vorab im asiatischen Raum. Wenn hier ein Produzent ausfällt -

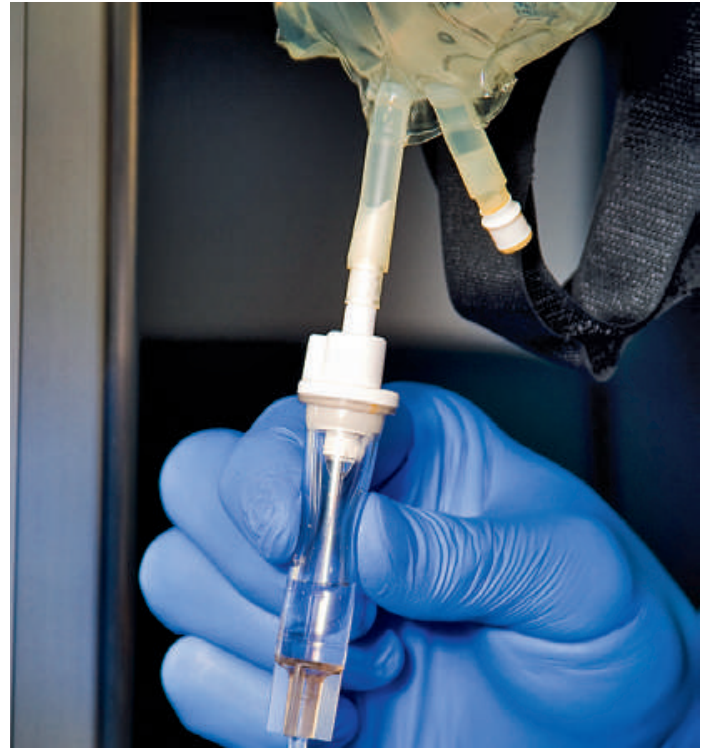

Immer öfter gibt es bei intravenösen Krebsmedikamenten Lieferprobleme.

Schattenseiten sind die fehlenden Lagerbestände und damit Pufferkapazität und Versorgungssicherheit. Steigt die Nachfrage unerwartet rasch, so ist hier keine genügende Kapazität vorhanden, um die Angebotsseite rasch zu bedienen. Gerade auch aufstrebende grosse Länder in Asien und Südamerika sowie im Osten Europas haben mehr Bedarf nach Medikamenten, die sie sich bis vor kurzem noch nicht leisten konnten.

\section{Schattenseiten sind die fehlenden Lagerbestände und damit fehlende Pufferkapazität und Versorgungssicherheit.}

aus welchen Gründen auch immer - oder auch nur in Lieferengpässe kommt, so sind sehr rasch viele oder alle Anbieter von Generika rund um den Globus betroffen. Alternativen gibt es dann keine mehr. Kurzfristiger Ersatz ist nicht möglich, da auch stillgelegte Fabriken nicht kurzfristig hochgefahren werden können angesichts der komplexen, zeit- und kostenintensiven regulatorischen Vorgaben für eine hochqualitative Medikamentenherstellung.

- Heute wird gezielt zeit- und bedarfsgerecht am Puls des Marktes kosteneffizient produziert. Die
- Qualität und Preis: Es wird moniert, dass die Generikapreise heute so unattraktiv seien, dass immer mehr das Interesse auf der Angebotsseite verloren gehe. Dies hat insbesondere dazu geführt, dass man in bestehende Produktionsanlagen nicht mehr investiert hat und diese wegen gravierender Qualitätsmängel teilweise behördlicherseits geschlossen werden mussten. Dies trifft speziell für die Herstellung intravenöser Formulierungen zu, da hier die Qualitätssicherung besonders anspruchsvoll ist. Dabei sei es auch zu Überreaktionen gekommen, z. B. dass man bei 
Symposium 23. August 2012, Kongress- und Kursaal Bern

Versorgungszugang und Finanzierung der innovativen, onkologischen Arzneimitteltherapie

Sicherstellung der medizinischen Versorgung bei zunehmender Verknappung von Medikamenten

Exorbitante Preise innovativer Krebsmittel und zunehmende Kosten in der Onkologie sind im Fokus der öffentlichen Diskussion angekommen. Der Bedarf an einigen wichtigen generischen Onkologika kann weltweit nicht mehr zuverlässig sichergestellt werden. Was steckt hinter dieser Problematik? Wie können Lösungen gefunden werden?

Weitere vielfältige Entwicklungen verändern nicht nur das medizinische Geschehen, sondern erfordern auch Weiterentwicklungen im Regulierungsbereich, sei es beim Marktzugang oder bei der Finanzierung durch die Sozialversicherungssysteme. Das Symposium greift diese vielfältigen Problematiken auf.

\section{Die Themenschwerpunkte}

- Aktueller Stand der onkologischen Arzneimitteltherapie

- Artikel $71 \mathrm{a} / \mathrm{b}$ KVV

- Zugang zur sicheren Versorgung in der Onkologie

- Beispiel «Onkologika»

- Von der Innovation bis zu Generika

- Praxisbeispiele

Vorsitz und Moderation

- Prof. Dr. med. Thomas Cerny, Präsident Krebsforschung Schweiz KFS, Vorstandsmitglied der Krebsliga Schweiz, Chefarzt Onkologie/Hämatologie, Kantonsspital St. Gallen

- Dr. rer. pol. Heinz Locher, Management + Consulting Services, Bern

Einführung: Prof. Dr. med. Jakob R. Passweg M. S., Präsident der Krebsliga Schweiz, Professor für Hämatologie, Chefarzt Hämatologie, Universitätsspital Basel

\section{Referenten}

- Jürg H. Schnetzer, Direktor Swissmedic

- Prof. Dr. pharm. Stefan Mühlebach, Spitalapotheker FPH, Chief Scientific Director, Vifor Pharma AG

- Andreas Faller, Vizedirektor und Leiter des Direktionsbereichs Kranken-, Unfallund Militärversicherung des Bundesamtes für Gesundheit BAG

- PD Dr. Stephan Schmitz, Berufsverband der niedergelassenen Hämatologen und Onkologen BNHO, Köln

- Dr. Fabrizio Guidi, Country Manager, Sanofi-Aventis (Schweiz) AG

- Jacques-Henri Weidmann, Director Market Access, Sanofi-Aventis (Schweiz) AG

- Dr. pharm. Enea Martinelli, Chefapotheker, Spitäler Frutigen, Meiringen, Interlaken

- Dr. med. Beat Seiler, Chef-Vertrauensarzt Helsana

- Dr. med. Jürg Nadig MAE, Präsident der Schweizerischen Gesellschaft für Medizinische Onkologie

- Andreas Bosshard, General Manager, Mepha (Teva) Schweiz

- Prof. Dr. med. Dieter Conen, Präsident Stiftung für Patientensicherheit

- Dr. med. Andreas Roos, Mitglied der Geschäftsleitung Sanitas

- Margrit Kessler, Nationalrätin, Patientenvertretung

Das Symposium ist mit 6 Credits als Kernfortbildung der Schweizerischen Gesellschaft für Medizinische Onkologie SGMO und für alle übrigen med. Teilnehmenden als erweiterte Fortbildung anerkannt.

Detaillierte Informationen und Anmeldung unter www.rsmedicalconsult.com $\rightarrow$ Veranstaltungen.

Der Schweizerische Ärzteverlag ist Medienpartner des Symposiums. beanstandeten Qualitätsmängeln nicht angemessen fokussiert reagiert habe, sondern gleich die ganzen Fabriken stillgelegt habe.

- Als weiterer Grund wird z.B. im New England Journal of Medicine ein interessanter Punkt aufgegriffen, nämlich dass hier auch kalkulierte Engpässe im Spiel sein könnten, und zwar da, wo lukrative Alternativen für bewährte, aber generische Medikamente vorliegen. Dies wird natürlich rasch dort vermutet, wo Originalpräparatehersteller, auch Generikahersteller, mit einer hohen Marktmacht sind. Dass dies von den Firmen energisch bestritten wird, ist nicht unerwartet.

- Auch die übliche Verhaltenspsychologie bei Engpässen spielt wohl eine relevante Rolle, nämlich dass die Verbraucher zu horten beginnen und somit die prekäre Versorgungslage noch verschlimmern. Unsere Spitalapotheker stellen auch fest, dass die früher offenbar kulante, frühzeitige Informationspolitik der Pharmalieferanten bei zu erwartenden Lieferschwierigkeiten heute nicht mehr praktiziert wird, und sie sich mehrheitlich einfach kurzfristig vor kaum mehr korrigierbare Tatsachen gestellt sehen.

- Selten verkaufte, aber durchaus sehr wichtige Substanzen werden von den Herstellern aus reinen Kostenüberlegungen zunehmend aus dem Sortiment genommen. Man verweist auf umliegende Länder, wo solche Medikamente im Sortiment bleiben und über Vertriebskanäle beschafft werden können. Hier ist auch der Regulator gefordert, dass er solche Medikamente so behandelt, dass sie bei geringem Aufwand auch in unserem Land verfügbar bleiben.

\section{Was sind mögliche Folgen? Was können wir tun?}

Vorerst sind es tägliche Feuerwehrübungen der findigen Spitalapotheker, die im Beschaffungsdschungel einen gangbaren Weg für unsere Patienten finden müssen. Explizit und implizit wird aber immer mehr auch ein ungeplanter Alternativweg beschritten, indem Medikamentensortimente oder Therapiepläne kurzfristig umgestellt werden müssen, ohne dass dies in der Auswirkung auf die Patienten und die Kostenfolgen wirklich diskutiert wird. Wir wissen nicht, wie viele Patienten bei uns bereits zu Schaden gekommen sind durch verzögerte verfügbare oder inferiore Therapiealternativen oder ausgebliebene Behandlungen, da dies bisher nicht erfasst wurde.

Es ist nun zwingend und dringend, dass diese Situation auf der politischen Bühne Resonanz erzeugt und das BAG mit Swissmedic die Führerschaft übernimmt, um die landesweite Sicherstellung der Medikamentenversorgung für lebenswichtige Präparate anzupacken.

Literatur auf Anfrage beim Verfasser 